Research Article

\title{
Synchrotron Radiation X-Ray Absorption Spectroscopy and Spectroscopic Ellipsometry Studies of InSb Thin Films on GaAs Grown by Metalorganic Chemical Vapor Deposition
}

\author{
Yingda Qian, ${ }^{1,2}$ Yuanlan Liang, ${ }^{1,2}$ Xuguang Luo, ${ }^{1,2}$ Kaiyan He ${ }^{(D)},{ }^{1,2}$ Wenhong Sun $(D)$, \\ Hao-Hsiung Lin, ${ }^{3}$ Devki N. Talwar, ${ }^{4,5}$ Ting-Shan Chan, ${ }^{6}$ Ian Ferguson, ${ }^{7}$ Lingyu Wan, ${ }^{1,2}$ \\ Qingyi Yang, ${ }^{1,2}$ and Zhe Chuan Feng $\mathbb{D D}^{1,2}$ \\ ${ }^{1}$ College of Physical Science and Technology, Guangxi University, Nanning 530004, China \\ ${ }^{2}$ Laboratory of Optoelectronic Materials and Detection Technology, Guangxi Key Laboratory for the Relativistic Astrophysics, \\ Guangxi University, Nanning 530004, China \\ ${ }^{3}$ Graduate Institute of Electronics and Department of Electrical Engineering, National Taiwan University, \\ Taipei 106-17, Taiwan \\ ${ }^{4}$ Department of Physics, Indiana University of Pennsylvania, Indiana, PA 15705-1087, USA \\ ${ }^{5}$ Department of Physics, University of North Florida, Jacksonville, FL 32224, USA \\ ${ }^{6}$ National Synchrotron Radiation Research Center, Hsinchu 300-76, Taiwan \\ ${ }^{7}$ Department of Electrical and Computer Engineering, Missouri University of Science and Technology, Rolla, MO 65409, USA
}

Correspondence should be addressed to Kaiyan He; gredhky@gxu.edu.cn, Wenhong Sun; youzi7002@gxu.edu.cn, and Zhe Chuan Feng; fengzc@gxu.edu.cn

Received 22 January 2018; Revised 6 April 2018; Accepted 29 April 2018; Published 8 July 2018

Academic Editor: Si Joon Kim

Copyright (C) 2018 Yingda Qian et al. This is an open access article distributed under the Creative Commons Attribution License, which permits unrestricted use, distribution, and reproduction in any medium, provided the original work is properly cited.

\begin{abstract}
A series of ultrathin InSb films grown on GaAs by low-pressure metalorganic chemical vapor deposition with different $\mathrm{V} / \mathrm{III}$ ratios were investigated thoroughly using spectroscopic ellipsometry (SE), X-ray diffraction, and synchrotron radiation X-ray absorption spectroscopy. The results predicted that InSb films on GaAs grown under too high or too low V/III ratios are with poor quality, while those grown with proper V/III ratios of 4.20-4.78 possess the high crystalline quality. The temperature-dependent SE $\left(20-300^{\circ} \mathrm{C}\right)$ and simulation showed smooth variations of SE spectra, optical constants $\left(n, k, e_{1}\right.$, and $\left.\varepsilon_{2}\right)$, and critical energy points $\left(E_{1}, E_{1}+\Delta_{1}, E_{0}^{\prime}, E_{2}\right.$, and $\left.E_{1}^{\prime}\right)$ for InSb film when temperature increased from $20^{\circ} \mathrm{C}$ to $250^{\circ} \mathrm{C}$, while at $300^{\circ} \mathrm{C}$, large changes appeared. Our study revealed the oxidation of about two atomic layers and the formation of an indium-oxide (InO) layer of $\sim 5.4 \mathrm{~nm}$. This indicates the high temperature limitation for the use of InSb/GaAs materials, up to $250^{\circ} \mathrm{C}$.
\end{abstract}

\section{Introduction}

As a member of the III-V compound semiconductors family, the growth of ultrathin films of indium antimonide has attracted a great deal of attention for its use in midwavelength infrared detectors (viz., thermal imaging cameras and forward looking infrared systems), magnetic sensors, magnetoresistors, field-effect transistors, photoconductors, and high-speed electronic devices [1-33]. Among other semiconductors, the intrinsic binary InSb possesses the highest electron mobility $\left(\sim 7.8 \times 10^{4} \mathrm{~cm}^{2} / \mathrm{V} \cdot \mathrm{s}\right)$, higher breakdown field $\left(\sim 10^{3} \mathrm{~V} / \mathrm{cm}\right)$, high saturated electron drift velocity $\left(\sim 5 \times 10^{7} \mathrm{~cm} / \mathrm{s}\right)$, small effective mass $\left(m_{\mathrm{e}}^{*} \sim 0.013 m_{\mathrm{o}}\right)$, lowest energy band gap $E_{g} \sim 0.18 \mathrm{eV}$ (at $300 \mathrm{~K}$ ), and ballistic length up to $\sim 0.7 \mu \mathrm{m}$ (at $300 \mathrm{~K}$ ). The InSb material can be used as a lattice-matched substrate for epitaxial growth of CdTe [34] and other relevant heterostructures and superlattices [35]. The InSb-based ternary as well as quaternary alloys are equally valuable for realizing midwavelength infrared detectors [36-39]. More recently, there has been a growing challenge to explore the possibility of developing InSb-based quantum dots and nanostructured laser diodes [40-42] for optoelectronic as well as photonic device 
applications in the 3-5 $\mu \mathrm{m}$ wavelength range for room temperature operations.

In optoelectronic devices, one must grow ultrathin layers of InSb on semi-insulating infrared transparent substrates to prevent current leakage. While CdTe is the only semiinsulating lattice-matched substrate available for InSb growth, it is difficult, however, to avoid the formation of the $\mathrm{In}_{2} \mathrm{Te}_{3}$ precipitates at the InSb/CdTe interface $[43,44]$. Hence, many alternative materials (viz., Si, GaAs, InP, sapphire, and mica) have been chosen as substrates for preparing InSb epifilms [2, 15-17, 19-32] by molecular beam epitaxy, liquid-phase epitaxy $[18,30]$, metalorganic chemical vapor deposition (MOCVD) [20-22, 31], metalorganic magnetron sputtering [32, 45], and two-step growth process [46] methods. Despite a large (14.6\%) lattice mismatch between InSb and GaAs, the semi-insulating GaAs is considered as an attractive substrate due to high chemical stability and resistivity. Efforts have been made to grow InSb thin films on four-inch GaAs substrates [31, 32, 47]. Also many attempts made by MOCVD have shown improvements in InSb film growth on large area semi-insulating GaAs substrates [47-49]. These efforts have certainly helped provided the increasing mass production needs of isolated $\mathrm{InSb} / \mathrm{GaAs}$ (001) thin films for integration with III-V semiconductor platform to achieve better performance of both the electronic and photonic devices.

However, the incompatibility of the lattice constants between the two materials strongly limits the quality of InSb epilayers grown on GaAs [23]. In general, the epifilms suffer from high density of dislocations or defects near the film/substrate interface. These defects propagating throughout the entire material create antiphase domains and cause autodoping effects. The layers with intrinsic defects may also affect the carrier mobility and leakage current in both the electronic and photonic devices. To circumvent these difficulties and improve the crystallinity of InSb films, several strategies are pursued-especially in optimizing the growth conditions by controlling the V/III ratios, pressure, growth temperature, growth rate, and film thickness [20-22, 31, 47, 48].

Accurate estimation of InSb epifilm thickness is of paramount importance using them for device engineering. Traditionally, the thin semiconducting films are characterized by using Hall measurements, XRD, ultraviolet-visible spectrophotometry, cross-sectional scanning electron microscopy [20, 21, 23, 49, 50], and so on. Being destructive, most of these methods are not convenient for assessing material quality required in electronic industry for device productions. Due to the narrow band gap of InSb, no reflectance interference fringes appear in the ultraviolet-visible wavelength range from the InSb/GaAs heterostructure. Therefore, a variety of nondestructive and penetrative tools have been exploited, providing nanoscale resolution for evaluating film thickness with greater degree of accuracy. Some of these techniques used in characterizing large area wafers include the atomic force microscopy, energy-dispersive $\mathrm{X}$-ray spectroscopy, secondary-ion-mass spectrometry, and SE. RSS is another valuable and nondestructive tool to offer useful information on the crystalline quality and other
TABLE 1: Thicknesses and MSE of InSb thin film samples from SE.

\begin{tabular}{lccccc}
\hline Sample no. & S1 & S2 & S3 & S4 & S5 \\
\hline V/III ratio & 3.91 & 3.98 & 4.20 & 4.78 & 5.38 \\
Layer thickness (nm) & 56.4 & 63.5 & 52.0 & 34.3 & 51.4 \\
MSE & 7.20 & 6.14 & 4.58 & 5.90 & 3.82 \\
\hline
\end{tabular}

parameters necessary for optimizing the InSb epifilm growth [31].

In this study, we present the results of our comprehensive investigations using SE, XRD, and SR-XAS, respectively, to assess the InSb film thickness and report the effects of V/III source ratios on the films crystalline quality for optimizing the MOCVD growth parameters, which were not exactly achieved from previous reports [31]. The SR-XAS was used to acquire the structural properties of the material at the atomic scale. Furthermore, temperature-dependent optical constants of InSb thin films between $25^{\circ} \mathrm{C}$ and $300^{\circ} \mathrm{C}$ $(n, k$, and $\varepsilon$ ) were probed by exploiting SE. This work provides a helpful guide to thin film characterization procedures required to monitor the growth processes, understanding the chemical and physical properties of materials and guiding the designs of high-performance InSb thin film devices.

\section{Experimental}

2.1. Material Growth. The growth of ultrathin uniform InSb thin epilayers was carried out on 4-inch semi-insulating (SI) GaAs (100) substrates using a low-pressure MOCVD method in vertical configuration by exploiting a high-speed rotating disk (180 $\mathrm{mm}$ diameter) reactor [31, 47]. The substrate orientation of 4 -inch SI GaAs (100) was $2-4^{\circ}$ off towards $\langle 110\rangle$. The trimethyl indium and trimethyl antimony sources were used as vapor-phase constituents of In and $\mathrm{Sb}$ into a reaction chamber at approximately $\sim 20^{\circ} \mathrm{C}$ and with the bubbler pressures set at 400 Torr for trimethyl indium and 323 Torr for trimethyl antimony, respectively. Hydrogen acted as carrier gas, and the pyrometer growth temperature was $395^{\circ} \mathrm{C}$. In this paper, we have studied a series of five $\mathrm{InSb} / \mathrm{GaAs}$ samples grown with different V/III source flow ratios and thickness. Table 1 summarizes the growth relevant parameters of the samples used from the MOCVD growth [31] and their thicknesses determined in this study. For InSb growth using trimethyl indium and trimethyl antimony, the surface morphology was found to be very sensitive to the V/III ratio and closely reflected the crystallinity of as-grown films. All InSb films with V/III ratio varied between 3.91 and 5.38 exhibit mirror-like surface morphology having thickness ranging from 34 to $64 \mathrm{~nm}$. To examine the effects of V/III ratio on the InSb film characteristics, we named the five samples $S 1$ to $\mathrm{S} 5$ in the order of V/III ratio corresponding to their growth run numbers (IA376, 380, 373, 370, and 371, resp. [31]).

\subsection{Optical Measurements}

2.2.1. Spectroscopic Ellipsometry. SE is a valuable nondestructive technique commonly used for determining 


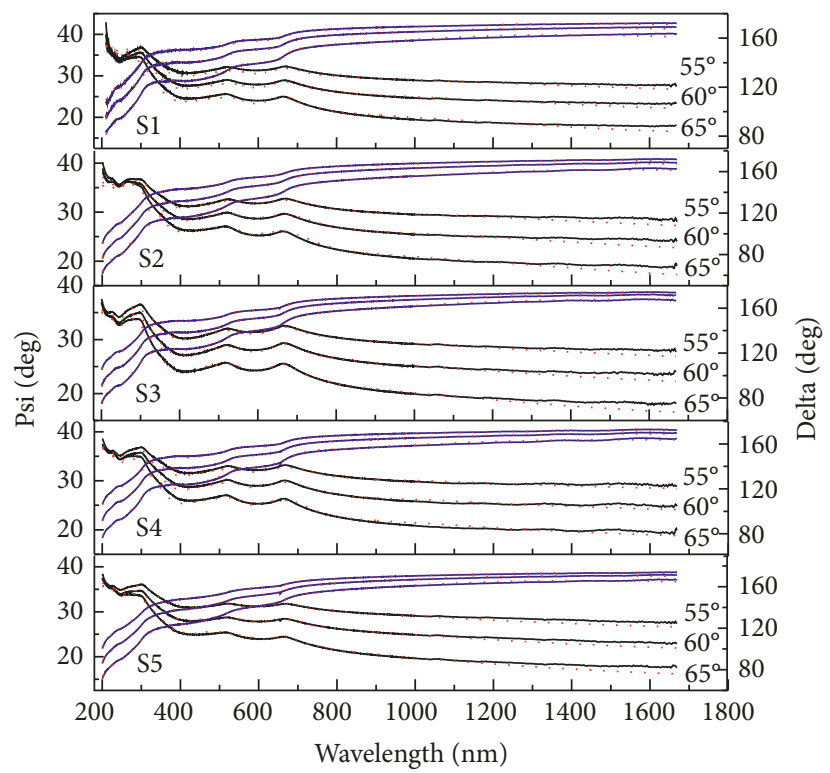

FIgURE 1: Experimental SE data and model fitting for five MOCVD grown InSb/GaAs samples.

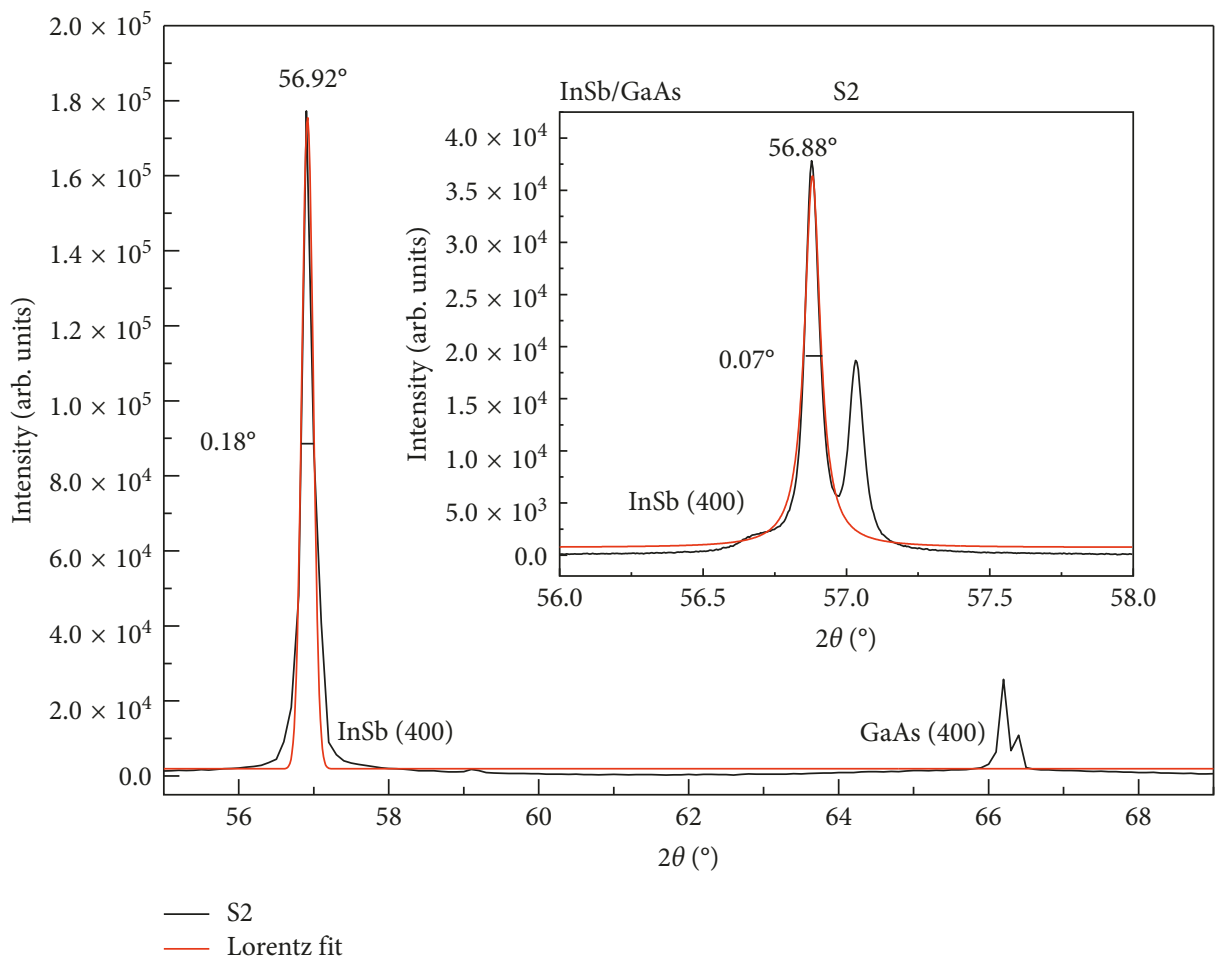

FIGURE 2: XRD spectrum and Lorentz fit of S2 in the angle range of $55^{\circ}-69^{\circ}$.

thickness, optical constants of materials; the method has also been employed for a complete depth profiling in semiconducting epitaxially grown ultrathin or thin films. While the conventional SE approach suffers from the drawback of slow data acquisition process and covers a limited spectral range, the phase-modulated method that we have employed here offers the fast and precise data acquisition over a large wavelength range. A dual rotating-compensator Mueller matrix ellipsometer (ME-L ellipsometer, Wuhan Eoptics
Technology Co. Ltd., China), equipped with Linkam Scientific heating and cooling stage device (THMSG600E), was used for the acquisition of ellipsometric spectra on ultrathin InSb epifilms. At room temperature, we acquired (Figure 1) two parameters $\Psi$ and $\Delta$, represented the ratio of amplitude decay between and $p$ and $s$ polarization of the reflected light and their phase difference, respectively, as a function of wavelength $(\lambda)$ from $200 \mathrm{~nm}$ to $1670 \mathrm{~nm}$ at $1 \mathrm{~nm}$ step (or energy from $0.74 \mathrm{eV}$ to $6.2 \mathrm{eV}$ ) at three angles of incidence $55^{\circ}, 60^{\circ}$, and $65^{\circ}$. 
TABLE 2: The fitting and calculation results from XRD of five InSb samples with different V/III ratios.

\begin{tabular}{|c|c|c|c|c|c|}
\hline Sample no. & S1 (376) & S2 (380) & S3 (373) & S4 (370) & S5 (371) \\
\hline V/III ratio & 3.91 & 3.98 & 4.2 & 4.78 & 5.38 \\
\hline XRD FWHM $(\Delta 2 \theta$, degree $)$ & 0.21 & 0.07 & 0.05 & 0.11 & 0.1 \\
\hline Peak position ( $2 \theta$, degree $)$ & 56.95 & 56.92 & 56.92 & 56.96 & 56.96 \\
\hline Layer thickness (nm) (SE measured) & 56.4 & 63.5 & 52.0 & 34.3 & 51.4 \\
\hline Calculated $\beta(\Delta 2 \theta$, degree $)$ & 0.29 & 0.26 & 0.32 & 0.48 & 0.32 \\
\hline
\end{tabular}

2.2.2. Raman Scattering Spectroscopy. RSS is a powerful and nondestructive technique for providing valuable information on materials' characteristics-especially for assessing epilayer thickness, strain, disorder, and site selectivity of defects. The RSS method is particularly suited for probing the local atomic and/or nanoscale structural changes in the InSb ultrathin films grown on SI GaAs while making the careful analysis of its subtle spectral variations. At the earlier time, we had quickly measured RSS on our InSb samples using a Raman spectrometer with the excitation from a HeNe laser $633 \mathrm{~nm}$ line. Later on, after more years, we performed further Raman measurements using 514-nm laser excitation on these samples, with similar results obtained. To avoid duplication, these data were not presented here.

2.2.3. X-Ray Diffraction Spectroscopy. As a common technique for material characterization, the XRD is widely used to evaluate the quality of crystal structure. It is sensitive for estimating stress/strains in epitaxially grown thin films. Furthermore, the peak position and the full width at half maximum (FWHM) of X-ray diffraction spectra give the information of crystal orientation and crystal quality. The diffractometer we used in experiments is Rigaku MiniFlex 600 , Japan. The five InSb samples were measured by this $\mathrm{X}$-ray diffractometer using a $\mathrm{Cu} \mathrm{K} \alpha$ radiation $(\lambda=1.5406 \AA)$.

\subsection{Synchrotron Radiation X-Ray Absorption Spectroscopy.}

XAFS spectra were collected for the InSb thin film samples in $\mathrm{X}$-ray fluorescence yield mode at the beam line $01 \mathrm{C} 1$ of the National Synchrotron Radiation Research Center in Hsinchu, Taiwan. The photon energy for the XAFS covered the range from 27,640 to $28,840 \mathrm{eV}$. The intensity of X-ray was monitored by a liquid $\mathrm{N}_{2}$-filled ionization chamber, and fluorescence emitted from the sample was measured by an argon-filled Stern-Held-Lytle detector. A Si (111) doublecrystal monochromater with a $0.5-\mathrm{mm}$ entrance slit was used. A filter was inserted between the sample and the detector window to reduce the noise from scattering and to improve the spectrum quality. The incident photon direction was 45 degree to surface of the sample, and the flux $I_{0}$ of incident photon was monitored simultaneously by an ion chamber located just before the sample chamber. All measurements were made at RT, and all X-ray absorption spectra were normalized to $I_{0}$.

\section{Results and Discussion}

3.1. Spectroscopic Ellipsometry at Room Temperature. The measured SE spectra are fitted empirically by minimizing the
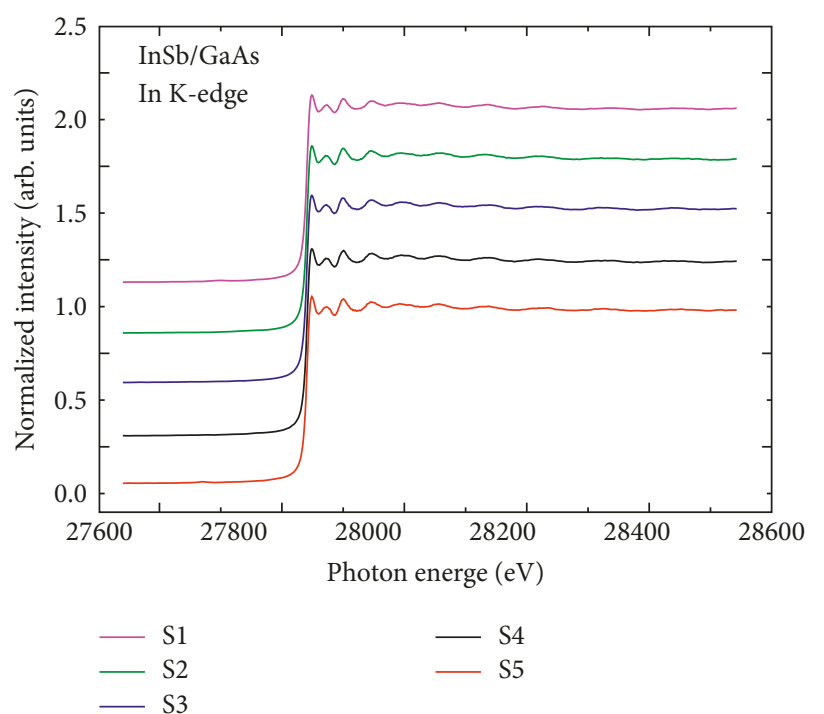

FIgURE 3: X-ray absorption spectra of In-K edge $(27,940 \mathrm{eV})$ for five InSb samples.

squared differences between the observed and calculated $\Psi$ and $\Delta$ values, which generated from the fitting model at the corresponding wavelengths. The quality of the fit can be judged by the mean square error (MSE) defined as follows:

$$
\text { MSE }=\frac{1}{2 n-m} \sum_{i}^{n}\left[\left(\frac{\Psi_{i}^{\bmod }-\Psi_{i}^{\exp }}{\sigma_{\Psi, i}^{\exp }}\right)^{2}+\left(\frac{\Delta_{i}^{\bmod }-\Delta_{i}^{\exp }}{\sigma_{\Delta, i}^{\exp }}\right)^{2}\right],
$$

where $n$ stands for the total number of experimental measurements of $\Psi$ and $\Delta$ for each chosen wavelength, $m$ represents the number of fitting parameters used, the superscripts "mod" and "exp" represent the appropriate values of model and experimental data, and $\sigma$ signifies the MSE between the calculated and experiment data at each wavelength.

In this study, the SE data were fitted using Tauc-Lorentz multiple oscillator modes [48]:

$$
\varepsilon_{2}(E) \begin{cases}=\frac{A E_{0} C\left(E-E_{g}\right)^{2}}{\left(E^{2}-E_{0}^{2}\right)^{2}+C^{2} E^{2}} \cdot \frac{1}{E}, & E>E_{g} \\ =0, & E \leq E_{g}\end{cases}
$$

where $E$ is photon transition energy, $A$ and $C$ are fitting constants, and estimates are obtained by fitting $\Psi$ and $\Delta$. Equation (2) is useful for evaluating the dielectric function $\varepsilon$ 


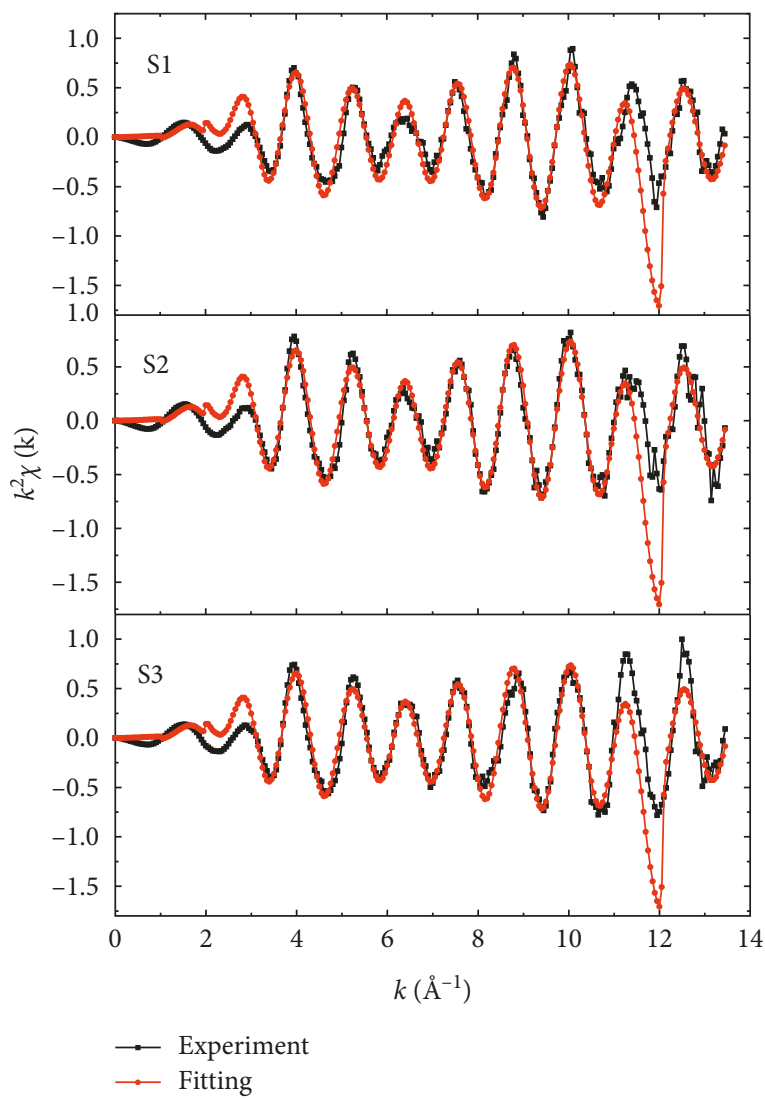

(a)

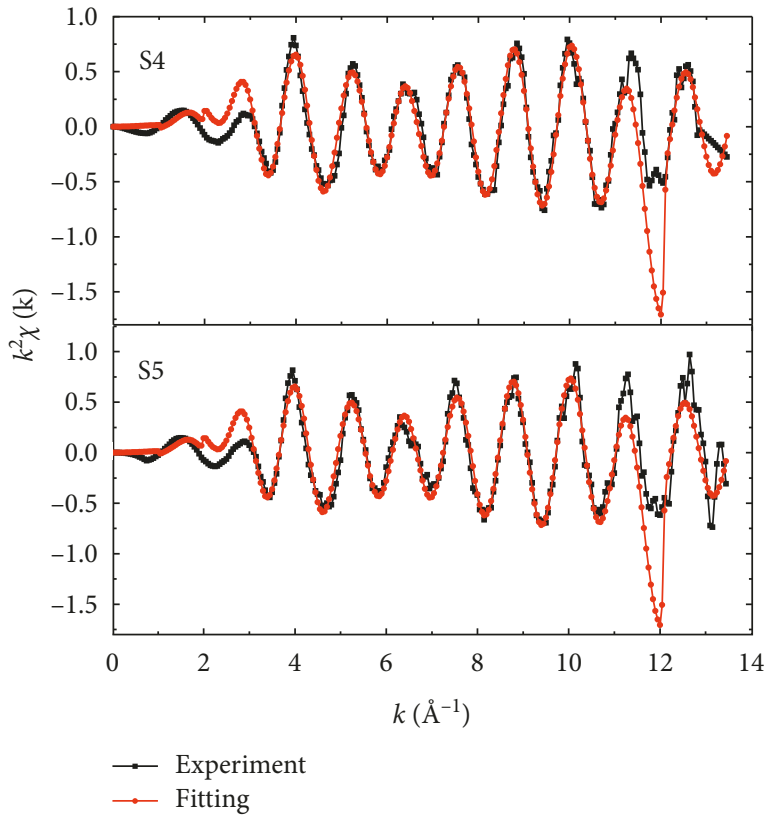

(b)

FIgURE 4: The In K-edge oscillation $k^{2} \chi(k)$ for all the measured InSb thin film samples.

(or $n, k$ ). The real part of the dielectric function $\varepsilon_{1}$ is obtained by exploiting the Kramers-Kronig integrations, that is,

$$
\varepsilon_{1}(E)=\varepsilon_{1}(\infty)+\frac{2}{\pi} P \int_{E_{g}}^{\infty} \frac{\xi \varepsilon_{2}(\xi)}{\xi^{2}-E^{2}} d \xi
$$

In (3), the term $P$ is the main part of the Cauchy integral, where $\varepsilon_{1}(\infty)$ is added as a fitting parameter.

Figure 1 shows experimental SE $\Psi$ and $\Delta$ spectra of five $\mathrm{InSb} / \mathrm{GaAs}$ samples, measured with three incident angels at room temperature (RT). The spectral oscillations of the five samples are similar, indicating that they have similar optical properties. All of the $\Psi$ and $\Delta$ data with variable angles were considered in the calculation of the optical parameters like $n$ and $k$. The thicknesses of InSb films were extracted through analyzing the SE data. The three-layer model, substrate/film/oxidation, or surface roughness was first tested for modeling. The simulation results showed the surface oxidation layer with the thickness of $0.01 \mathrm{~nm}$ or less for all sample. This is physically meaningless, that is, the thickness of the surface oxidation layer should be zero. Therefore, the two-layer model, substrate/film, was used for modeling on five InSb/GaAs samples here. Fitting results are listed in Table 1.

3.2. The X-Ray Diffraction Data and Fits. Figure 2 shows the wide-scan XRD spectrum of a typical InSb/GaAs sample (S2) in the angle range $(2 \theta)$ from $55^{\circ}$ to $69^{\circ}$ at $0.1^{\circ}$ step. The intensity of the GaAs (400) peak was observed to be weaker than that of the InSb (400) peak. This phenomenon was due to that the semi-insulating GaAs wafer cut $2-4^{\circ}$ off $(100)$ towards $<110>$ was used as the substrate. The surface of GaAs wafer was deviated from the real (100) orientation. The GaAs wafer was not the real GaAs (100) substrate or not with the real (100) orientation surface. This type of semi-insulating GaAs (100) $2^{\circ}$ off towards $<110\rangle$ substrate was successfully used for the growth of InSb thin films [47, 49]. During the growth process, it could lead the heteromismatch-induced dislocations to spread along the InSb/GaAs interface plane but not along the film growth normal direction, that is, not to the InSb film surface, so as to greatly decrease the dislocation density at the surface area of InSb film.

From this rough scan, the InSb (400) peak appeared as a single peak with the FWHM of $0.18^{\circ}$. Inset of Figure 2 shows fine-scan XRD spectrum in the angle $(2 \theta)$ of $56^{\circ}-58^{\circ}$ at $0.005^{\circ}$ step. The observation of the peak doublet splitting is caused due to the $\mathrm{X}$-ray source $\mathrm{Cu} \mathrm{K} \alpha_{1}$ and $\mathrm{Cu} \mathrm{K} \alpha_{2}$ radiation. The well-separation of the InSb (400) peak doublet and the narrow FWHM of $0.07^{\circ}$ from the InSb (400) $\mathrm{K} \alpha \alpha_{1}$ peak indicated good crystallinity of the film. A sharp diffraction peak was observed at $2 \theta=56.88^{\circ}$, which corresponds to the (400) crystalline plane of InSb (S2). The other diffraction peak located at $66.20^{\circ}$ can indexed to the (400) crystal plane of GaAs substrate. Table 2 lists the values of 


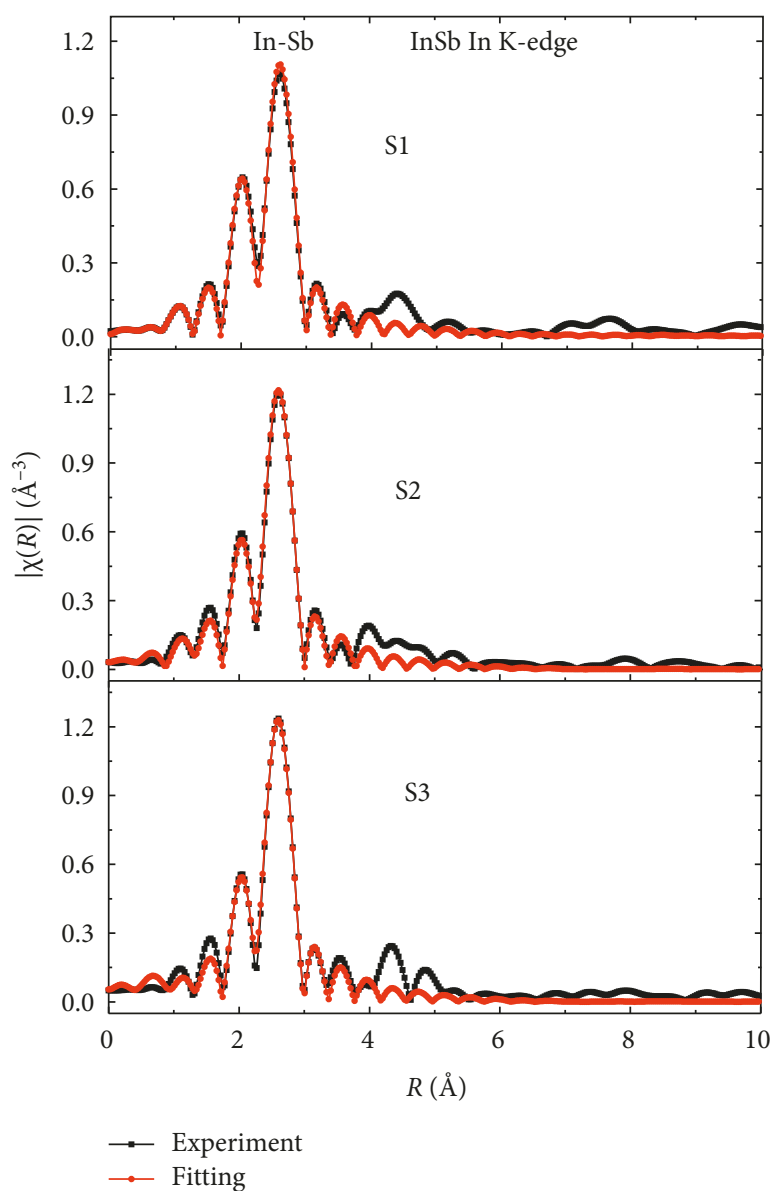

(a)

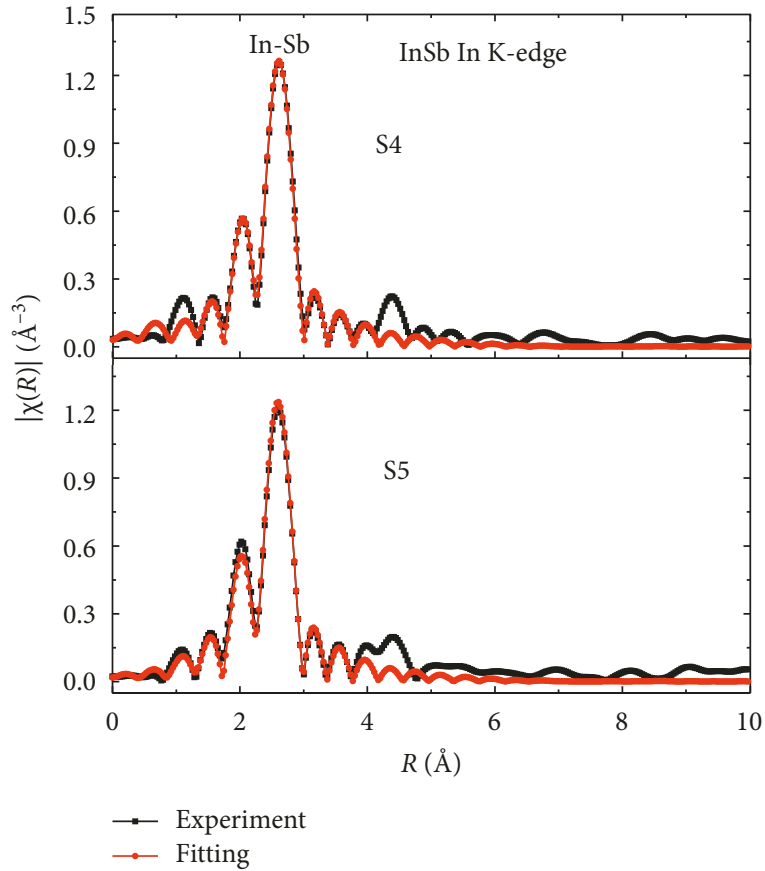

(b)

FIgURE 5: The magnitude of the Fourier transforms of measured samples (black lines) and the results of fitting (red lines).

XRD peak and FWHM obtained by Lorentz fitting, for five InSb samples with different V/III ratios.

Farag et al. [51] calculated the crystallite size $(D)$ of the InSb film grown on GaAs from the broadening of few XRD peaks using the Debye-Scherrer equation (4):

$$
D=\frac{K_{\mathrm{S}} \cdot \lambda}{\beta \cos \theta},
$$

where $\lambda$ is the $\mathrm{X}$-ray wavelength of $\mathrm{Cu} \mathrm{K} \alpha(0.15418 \mathrm{~nm}), \beta$ is the width of the peak at half maximum intensity for the thin film, $K_{\mathrm{S}}$ is the Scherrer constant of the order of unity ( 0.95 for powder and 0.89 for film), and $\theta$ is the corresponding Bragg's angle.

Usually, finite size gives broader peaks. The contribution of our InSb film thickness, $D$, to the XRD broad peak width, $\beta$, can be estimated by modified Debye-Scherrer formula (5),

$$
\beta=\frac{0.89 \lambda}{D \cos \theta} .
$$

The so-calculated $\beta$ values using the InSb film thickness obtained from SE are also listed in Table 2. It is seen that these $\beta$ values are much larger than the XRD FWHMs measured in our experiments. This predicts the high crystalline quality of our MOCVD grown InSb films on GaAs.
3.3. Simulations of SR-XAS Data. Indium K-edge extended $\mathrm{X}$-ray absorption fine structure (EXAFS) was employed to study the local structure of InSb thin films on GaAs. Figure 3 plots the four In K-edges EXAFS of InSb, with all of In absorption edges near $27,940 \mathrm{eV}$. Replicate EXAFS scans were coadded to improve the signal-to-noise ratio. Due to numerous diffraction peaks in the In-EXAFS spectra, the samples were spun to average out the diffraction peaks in the EXAFS spectra. All EXAFS spectra were collected to $1,200 \mathrm{eV}$ beyond the In K-edges. All EXAFS data were analyzed for wave vectors $(k)$ from $3.0 \AA^{-1}$ to $11.5 \AA^{-1}$. The chi data were $k^{2} \chi$ $(k)$ weighted as shown in Figure 4 and Fourier transformed with a window width of $k=0.5 \AA^{-1}$ to yield the R-space data. As shown in Figure 5, the simulated EXAFS spectra were generated, based on the documented crystallographic properties for In and Sb using ab initio-based theory.

We used ATHENA program to remove the background and extract the EXAFS oscillations from the k-space signals. To extract the bond length of $\mathrm{R}_{\mathrm{In}-\mathrm{Sb}}$, a structural model was built using the package IFEFFIT. Fitting results of the Fourier transforms show a good agreement with the measurement shown in Figure 5. With the first neighbor peak being well defined in the In absorption, we extracted the $R_{\mathrm{In}-\mathrm{Sb}}$ from the In data listed in Table 3. 
TABLE 3: The fitting results of the EXAFS data for first coordination shell atom around In atom.

\begin{tabular}{lccccc}
\hline Sample no. & V/III ratio & Amp & CN & $R_{\text {In-Sb }}(\AA)$ & $R$-factor \\
\hline S1 & 3.91 & $0.9500(0)$ & 3.80 & $2.8027(9)$ & $0.0052(5)$ \\
S2 & 3.98 & $0.9805(6)$ & 3.92 & $2.8044(9)$ & $0.0050(9)$ \\
S3 & 4.20 & $0.9807(3)$ & 3.92 & $2.8000(7)$ & $0.0089(1)$ \\
S4 & 4.78 & $0.9950(0)$ & 3.98 & $2.8014(3)$ & $0.0078(5)$ \\
S5 & 5.38 & $0.9898(6)$ & 3.96 & $2.7989(9)$ & $0.0055(2)$ \\
\hline
\end{tabular}

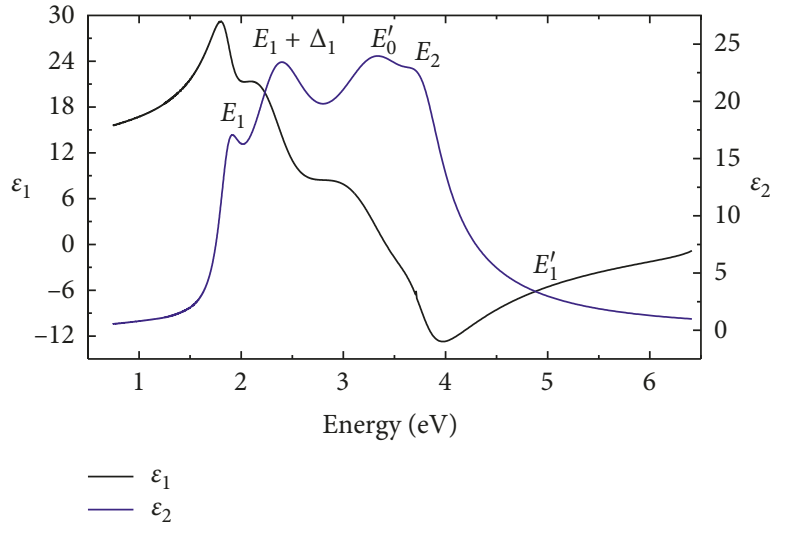

(a)

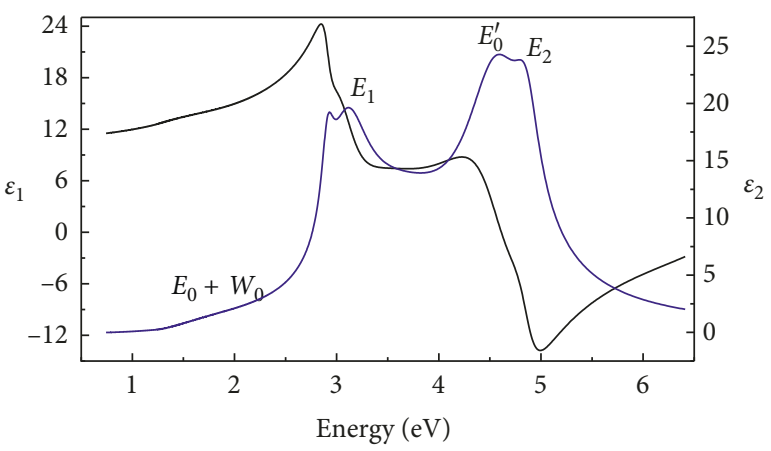

(b)

Figure 6: The dielectric functions of the bulk InSb and bulk GaAs.

Fitting results show that the bond length and coordination number of the first In-Sb shell are about $2.80 \AA$ and 4 , respectively. The bond length and the coordination number of unstrained InSb are $2.80 \AA$ and 4, respectively. The EXAFS fitting for five InSb/GaAs gave bond length values around $2.80 \AA$, and the sample $S 5$ with the highest V/III ratio of 5.38 has its $R_{\text {In-Sb }}$ slightly less than $2.80 \AA$. The coordination number $(\mathrm{CN})$ for all samples is close to but slightly below 4 (the regular value) with samples S4 and S5 nearest to 3.96-3.98, samples S2 and S3 at 3.92, and only sample S1 with the lowest V/III ratio of 3.91 significantly lower at 3.80. These variations could be caused by residual strain and too low/too high might cause higher lattice mismatch in the InSb/GaAs heterointerface, leading to higher residual strain within the epitaxial InSb film. This finding suggests that V/III ratios of 4.20 (S3) and 4.78 (S4) are the better growth parameter values, which is in consistent with the results from Raman scattering.

3.4. Further Analyses and Temperature-Dependent SE. The real part $\varepsilon_{1}$ and imaginary part $\varepsilon_{2}$ of the dielectric function of the bulk InSb and bulk GaAs as function of photon energy is shown in Figure 6. The peaks in these spectra reflect the high-energy-state transitions of bulk InSb and can be used to obtain several related critical energy points $\left(E_{1}, E_{1}+\Delta_{1}, E_{0}^{\prime}, E_{2}\right.$, and $\left.E_{1}^{\prime}\right)$ [52]. The high-energystate transitions of bulk GaAs are $E_{0}+W_{0}, E_{1}, E_{0}^{\prime}$, and $E_{2}$ [52]. Therefore, Figure 6 shows the peaks those are the highenergy-state transitions of the InSb film and none of GaAs substrate. D'Costa et al. [15] reported the SE study at room temperature for an $\mathrm{InSb} / \mathrm{GaAs}$ grown by molecular beam epitaxy, showing similar critical energy points from InSb.

From the simulation of the $\mathrm{SE} \Psi$ and $\Delta$ data in Figure 1, the reflective index $(n)$ and extinction coefficient $(k)$ versus wavelength or energy can be extracted. Figures $7(a)$ and $7(b)$ exhibit the comparative $n$ and $k$ variation with energy $(\mathrm{eV})$, for five InSb/GaAs, respectively. The critical energy points $\left(E_{1}, E_{1}+\Delta_{1}, E_{0}^{\prime}, E_{2}\right.$, and $\left.E_{1}^{\prime}\right)$ are marked as discussed in last paragraph.

Temperature-dependent (TD) SE measurements were performed in the temperature range from $20^{\circ} \mathrm{C}$ to $300^{\circ} \mathrm{C}$. Figure 8 presents a set of TD-SE experimental data for sample S2, which were measured at three different angles, $55^{\circ}, 60^{\circ}$, and $65^{\circ}$. Simulation fits were performed for all SE $\Psi$ and $\Delta$ data. As mentioned in Section 3.1 for RT-SE, the three-layer model-substrate/film/surface oxide-was first used for modeling. The best fitting simulation results showed the surface oxide layer with the thickness of $0.01 \mathrm{~nm}$ or less, that is, zero, for all SE data measured from $20^{\circ} \mathrm{C}$ to $250^{\circ} \mathrm{C}$. However, for the SE spectra ( $\Psi$ and $\Delta)$ data measured at $300^{\circ} \mathrm{C}$, the three-layer model-substrate/film/surface oxide-has to be used, resulting in a surface oxide thickness of $5.4 \mathrm{~nm}$ for sample S2. Other samples had similar results, that is, SE data between $\mathrm{RT}$ and $250^{\circ} \mathrm{C}$ showed no surface oxide layer but $\mathrm{SE}$ at $300^{\circ} \mathrm{C}$ revealed a near 5 -nm surface oxide layer, corresponding to about two atomic layer of indium oxides.

Figure 9 shows the variation of refractive index $n$ and extinction coefficient $k$ of InSb films in a temperature range from $20^{\circ} \mathrm{C}$ to $300^{\circ} \mathrm{C}$. As seen, the InSb film contains several absorption peaks in the wavelength range from 200 to 


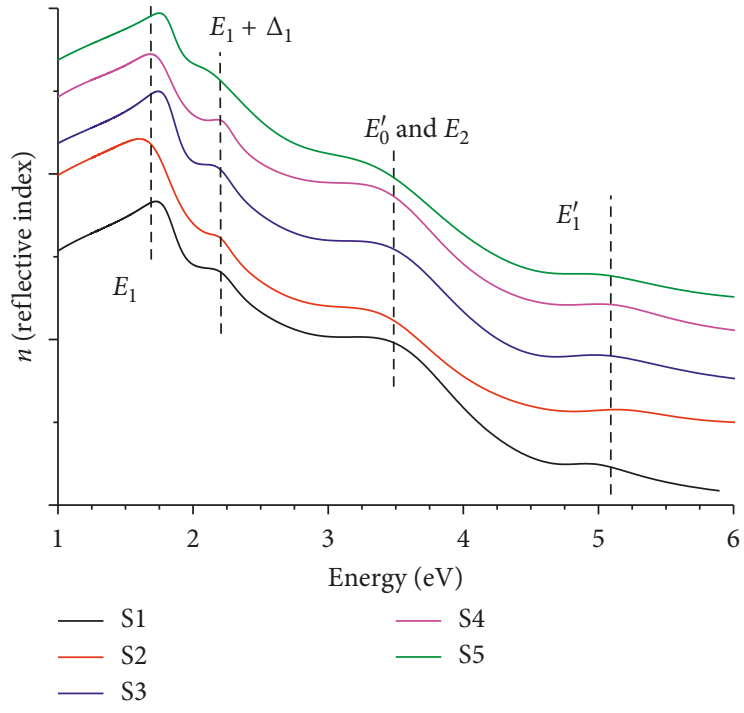

(a)

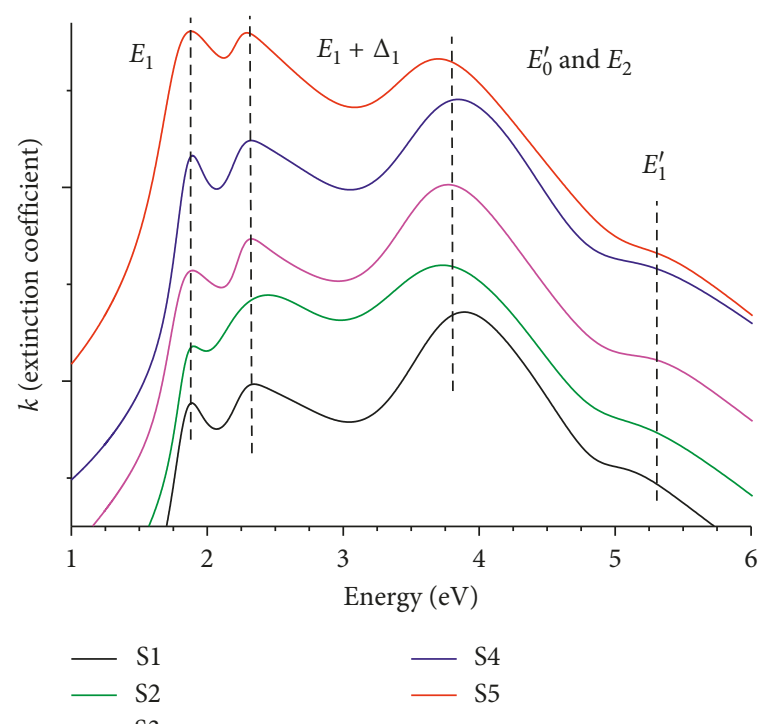

(b)

Figure 7: (a) Comparative reflective index $(n)$ and (b) comparative extinction coefficient $(k)$ for five InSb/GaAs samples, deduced from SE $\Psi$ and $\Delta$ data in Figure 1.

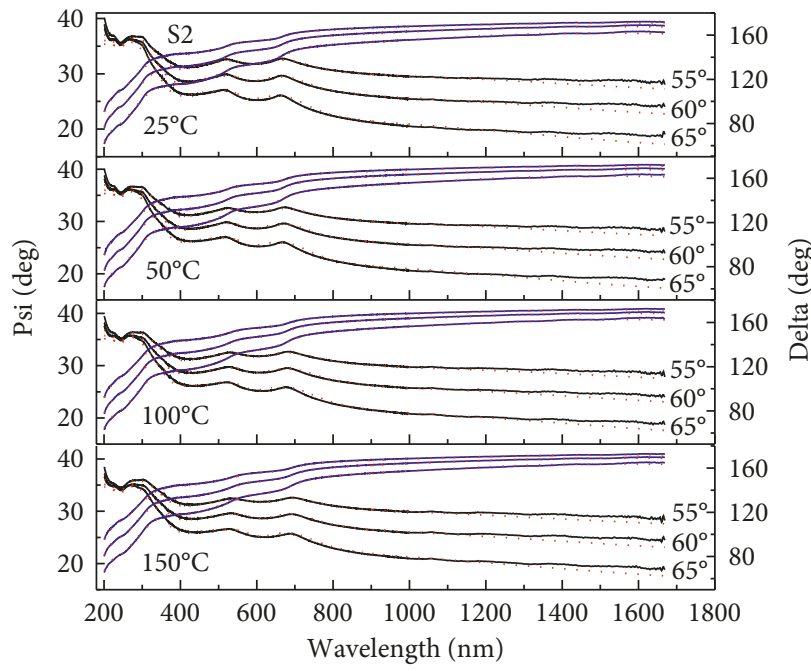

(a)

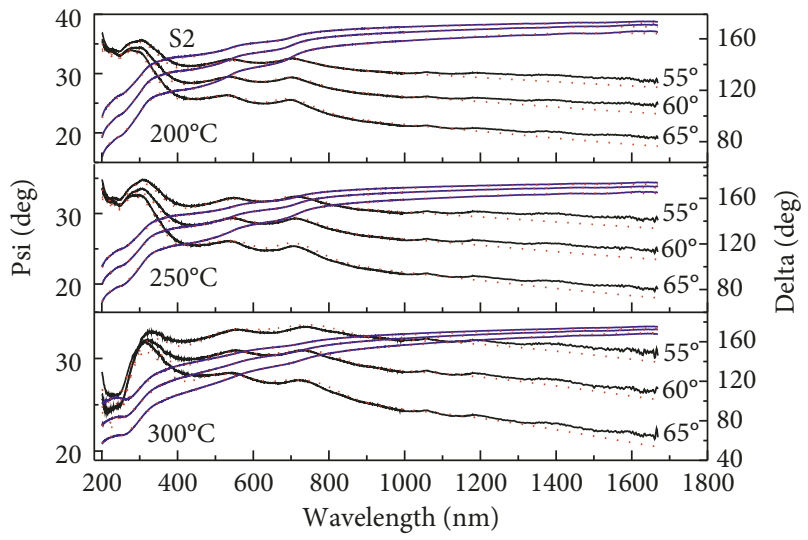

(b)

FIGURE 8: SE experimental data and model fitting for S2 at different temperatures.

$1500 \mathrm{~nm}$. Some absorption peaks shift to the longer wavelength (redshift) with increasing temperature. Kim et al. [53] performed TD-SE on bulk InSb over a temperature of $31 \mathrm{~K}$ to $675 \mathrm{~K}$. A similar redshift was observed in our experimental InSb films on GaAs over the temperature range of $20^{\circ} \mathrm{C}$ to $300^{\circ} \mathrm{C}$, that is, $293 \mathrm{~K}$ to $573 \mathrm{~K}$, comparable with that observed in other study [53].

Figure 10 exhibits the complex dielectric function, real and imaging parts, $\varepsilon_{1}$ and $\varepsilon_{2}$, calculated by $\varepsilon_{1}=n^{2}-k^{2}$ and $\varepsilon_{2}=2 n k$, of InSb film S2 with different temperatures. The high-energy critical points of $E_{1}, E_{1}+\Delta_{1}, E_{0}^{\prime}, E_{2}$, and $E_{1}^{\prime}$ are indicated in the figure. The gradual redshifts of these critical energy peaks with temperature increasing from $20^{\circ} \mathrm{C}$ to $250^{\circ} \mathrm{C}$ are seen. However, these redshifts of critical energy peaks in Figure 10 and $n$ and $k$ spectra in Figure 9 become much more pronounced as temperature increases to $300^{\circ} \mathrm{C}$. These large variations in $n$ and $k$ and $\varepsilon_{1}$ and $\varepsilon_{2}$ are due to the formation of an InO surface layer from the severe oxidation of the surface of InSb film at $300^{\circ} \mathrm{C}$. This oxidation could be ignored for InSb film measured in $20^{\circ} \mathrm{C}-250^{\circ} \mathrm{C}$ because the $\mathrm{SE}$ fits with three-layer model leaded to an InO surface thickness of $<0.01 \mathrm{~nm}$, much thinner than one monoatomic layer. But the oxide layer cannot be ignored in sample heated at $300^{\circ} \mathrm{C}$, that is, higher than $250^{\circ} \mathrm{C}$, the severe oxidation 


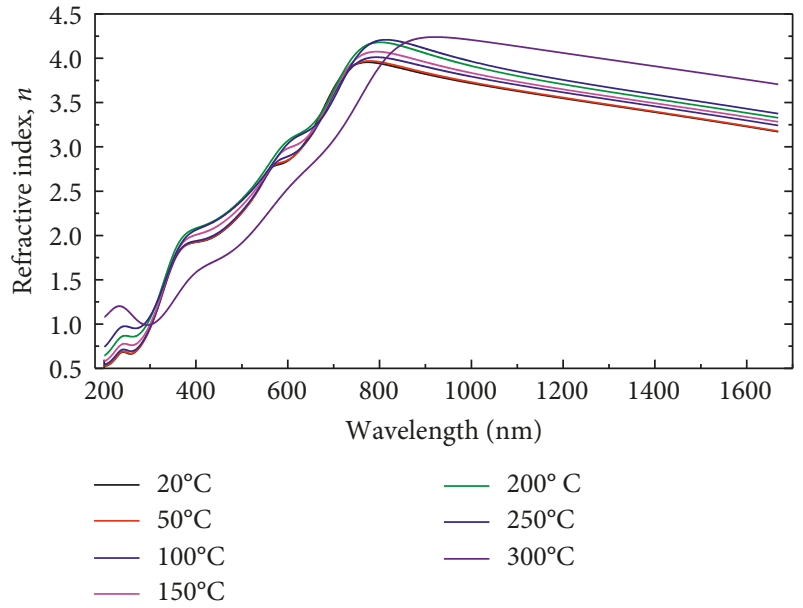

(a)

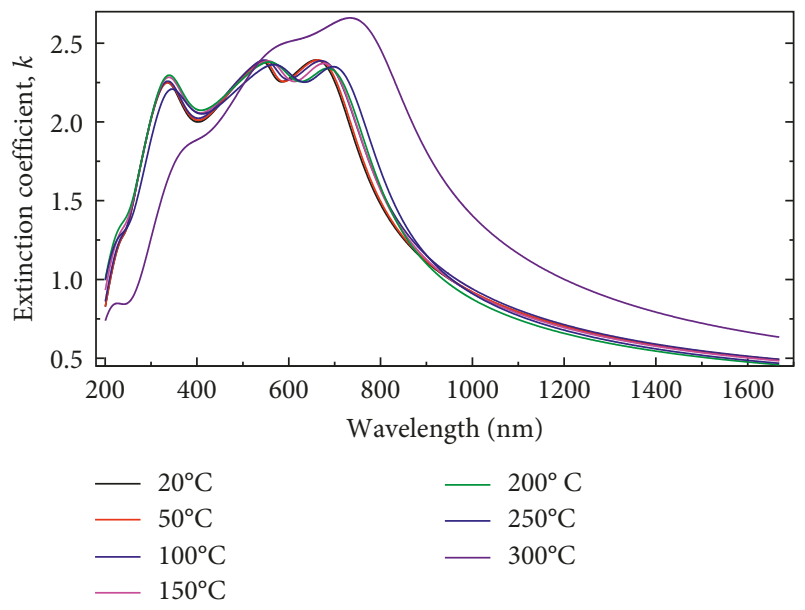

(b)

Figure 9: The refraction index $n$ (a) and the extinction coefficient $k$ (b) of InSb thin film (S2) with varied temperatures of $20^{\circ} \mathrm{C}-300^{\circ} \mathrm{C}$.

appeared on InSb, leading to the formation of a thin indiumoxide layer. This result may predicate the high temperature limitation for the use of InSb/GaAs materials.

\section{Conclusions}

In summary, a series of InSb thin films grown on 4-inch GaAs substrates by MOCVD technique, with different V/III ratios, were investigated by $\mathrm{SE}, \mathrm{XRD}$, and SR-XAS, respectively. InSb thin film thickness was extracted by fitting the experimental SE data. The crystallinity of the films was high quality extracted from the well separation of the InSb (400) peak doublet and the narrow FWHM of the InSb (400) $\mathrm{K} \alpha \alpha_{1}$ peak in the XRD spectrum. Through advanced synchrotron radiation technique of XAS and data simulation, the atomic scale bonding length and coordination number were obtained.

Through combined multiple technological analyses, the results showed that InSb films on GaAs grown under too high or too low V/III ratios are with poor quality, while those grown with proper V/III ratios of 4.20 and 4.78 possess the

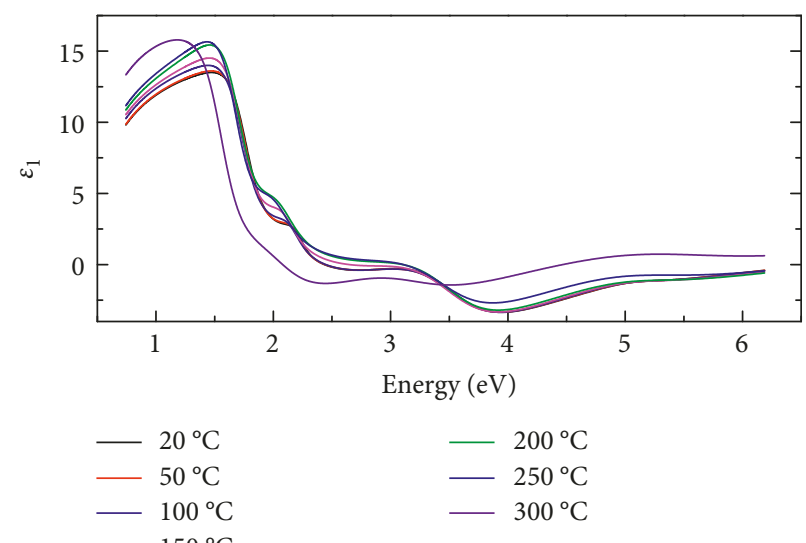

(a)

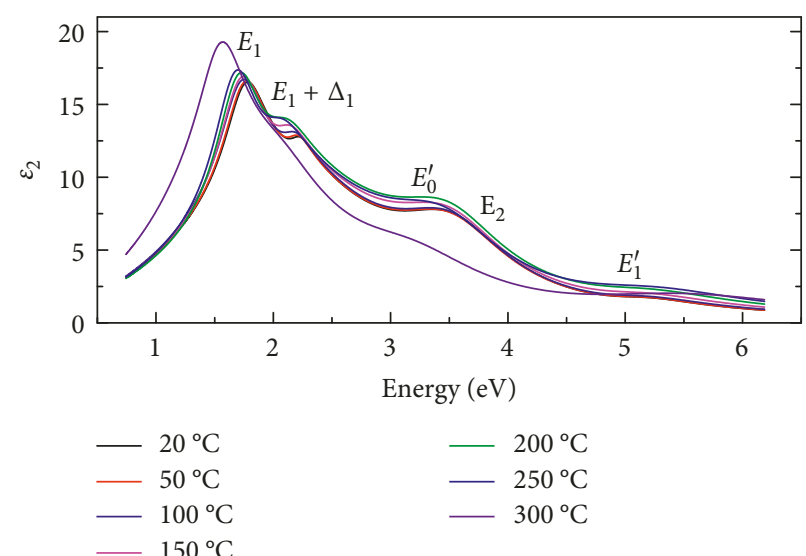

(b)

FIGURE 10: The real and imaginary part of the dielectric function $\varepsilon$ of the InSb thin film (S2) at varied temperatures of $20^{\circ} \mathrm{C}-300^{\circ} \mathrm{C}$.

high crystalline quality. These results are useful to the material growers for improving the growth processing.

The temperature-dependent (TD)-SE measurements $\left(20-300^{\circ} \mathrm{C}\right)$ and simulation revealed a significant phenomenon: the SE spectra, optical constants $\left(n, k, \varepsilon_{1}, \varepsilon_{2}\right)$, and critical energy points $\left(E_{1}, E_{1}+\Delta_{1}, E_{0}^{\prime}, E_{2}\right.$, and $\left.E_{1}^{\prime}\right)$ of the InSb thin films varied with temperature $(T)$ gradually and smoothly as $T$ increased from $20^{\circ} \mathrm{C}$ to $250^{\circ} \mathrm{C}$, and all SE spectra can be fitted well by the two-layer model (substrate/film). However, up to $300^{\circ} \mathrm{C}$, the SE spectra appeared to show large changes, and simulation revealed the existence of an indium-oxide ( $\mathrm{InO}$ ) layer of $\sim 5.4 \mathrm{~nm}$, that is, about two atomic layers. The optical constants $\left(n, k, \varepsilon_{1}, \varepsilon_{2}\right)$ and critical energy points $\left(E_{1}\right.$, $E_{1}+\Delta_{1}, E_{0}^{\prime}, E_{2}$, and $E_{1}^{\prime}$ ) had showed transilient changes from $250^{\circ} \mathrm{C}$ to $300^{\circ} \mathrm{C}$, which are due to the top about two atomic layers oxidized. This indicates the high temperature limitation for the use of InSb/GaAs materials, up to $250^{\circ} \mathrm{C}$, which provides a hinder to the device designers using InSb materials.

\section{Data Availability}

All types of data used to support the findings of this study are included within the article. 


\section{Conflicts of Interest}

The authors declare that there are no conflicts of interest regarding the publication of this paper.

\section{Acknowledgments}

This work was supported by National Natural Science Foundation of China (nos. 61367004 and 61504030) and special funding (nos. T3120097921 and T3120099202) for Guangxi distinguished professors (Bagui Rencai and Bagui Xuezhe). The authors acknowledge the support and help from Prof. Enwei Liang, Prof. Gu Xu, Prof. Jingli Chen, Ms. Qingxuan Li, Ms. Fangze Wang, Prof. Shiyuan Liu, and Dr. Chuanwei Zhang.

\section{References}

[1] Y. Cheng, J. Yang, Q. Jiang et al., "New insight to InSb-based thermoelectric materials: from the divorced eutectic design to remarkable high thermoelectric performance," Journal of Materials Chemistry A, vol. 5, no. 10, pp. 5163-5170, 2017.

[2] B. Wen Jia, K. Hua Tan, W. Khai Loke, S. Wicaksono, and S. F. Yoon, "Effects of surface reconstruction on the epitaxial growth of III-Sb on GaAs using interfacial misfit array," Applied Surface Science, vol. 399, pp. 220-228, 2017.

[3] M. Hilal, B. Rashid, S. Haider Khan, and A. Khan, "Investigation of electro-optical properties of InSb under the influence of spin-orbit interaction at room temperature," Materials Chemistry and Physics, vol. 184, pp. 41-48, 2016.

[4] D. Seo, J. Na, S. Lee, and S. Lim, "Behavior of GaSb (100) and InSb (100) surfaces in the presence of $\mathrm{H}_{2} \mathrm{O}_{2}$, in acidic and basic cleaning solutions," Applied Surface Science, vol. 399, pp. 523-534, 2017.

[5] A. Arlauskas, L. Subačius, A. Krotkus, and V.L. Malevich, "Terahertz emission from InSb illuminated by femtosecond laser pulses," Journal of Physics D: Applied Physics, vol. 50, no. 5, article 055101, 2017.

[6] R. Giulian, D. J. Manzo, J. B. Salazar et al., "Structural and electronic characterization of antimonide films made by magnetron sputtering," Journal of Physics D: Applied Physics, vol. 50, no. 7, article 075106, 2017.

[7] T. D. Subash, T. Gnanasekaran, C. Divya, and J. Jagannathan, "Design and simulated characteristics of nanosized InSb based heterostructure devices," Advances in Materials Science and Engineering, vol. 2014, Article ID 196732, 5 pages, 2014.

[8] L. Zhang, W. Tian, Q. Meng, M. Sun, N. Li, and Z. Lei, "Analysis on structural stress of $64 \times 64$ InSb IRFPAs with temperature dependent elastic underfill," Journal of Sensors, vol. 2014, Article ID 952323, 7 pages, 2014.

[9] J. Abautret, J. P. Perez, A. Evirgen, J. Rothman, A. Cordat, and P. Christol, "Characterization of midwave infrared InSb avalanche photodiode," Journal of Applied Physics, vol. 117, no. 24, article 244502, 2015.

[10] A. G. Hernández, Y. Kudriavtsev, S. Gallardo, M. Avendaño, and R. Asomoza, "Formation of self-organized nano-surfaces on III-V semiconductors by low energy oxygen ion bombardment," Materials Science in Semiconductor Processing, vol. 37, pp. 190-198, 2015.

[11] M. Shafa, H. Ji, L. Gao et al., "Mid-infrared photodetectors based on InSb micro/nanostructures grown on low-cost mica substrates," Materials Letters, vol. 169, pp. 77-81, 2016.
[12] E. B. Elkenany, "Optoelectronic and mechanical properties of InSb semiconductor under the effect of temperature," Silicon, vol. 8, no. 3, pp. 391-396, 2016.

[13] J. L. He, W. D. Hu, Z. H. Ye, Y. Q. Lv, X. S. Chen, and W. Lu, "Joint FDTD-optical/FEM-electrical numerical simulation of reflection-type subwavelength-microstructure insb infrared focal-plane arrays," Journal of Electronic Materials, vol. 45, no. 9, pp. 4552-4556, 2016.

[14] J. A. del Alamo, "Nanometre-scale electronics with III-V compound semiconductors," Nature, vol. 479, no. 7373, pp. 317-323, 2011.

[15] V. R. D’Costa, K. H. Tan, B. W. Jia, S. F. Yoon, and Y.-C. Yeo, "Mid-infrared to ultraviolet optical properties of InSb grown on GaAs by molecular beam epitaxy," Journal of Applied Physics, vol. 117, no. 22, article 223106, 2015.

[16] B. Wen Jia, K. Hua Tan, W. Khai Loke, S. Wicaksono, and S. F. Yoon, "Epitaxial growth of low threading dislocation density InSb on GaAs using self-assembled periodic interfacial misfit dislocations," Materials Letters, vol. 158, pp. 258-261, 2015.

[17] W. Sun, H. Fan, Z. Peng et al., "Photodiode properties of molecular beam epitaxial InSb on a heavily doped substrate," Infrared Physics \& Technology, vol. 62, pp. 143-146, 2014.

[18] Y. Sato, Y. Morita, and I. Kanno, "Performance estimation of InSb compound semiconductor detectors as a function of active area using alpha particles," Nuclear Instruments and Methods in Physics Research Section A: Accelerators, Spectrometers, Detectors and Associated Equipment, vol. 737, pp. 1-4, 2014.

[19] M. Mori, Y. Yasui, K. Nakayama, K. Nakatani, and K. Maezawa, "Effects of initial in coverage for preparation of InSb bilayer on electrical properties of InSb films grown by surface reconstruction controlled epitaxy," Japanese Journal of Applied Physics, vol. 51, no. 2, pp. 165-176, 2012.

[20] Y. J. Jin, D. H. Zhang, X. Z. Chen, and X. H. Tang, "Sb antisite defects in InSb epilayers prepared by metalorganic chemical vapor deposition," Journal of Crystal Growth, vol. 318, no. 1, pp. 356-359, 2011.

[21] S. Yamaguchi and M. Matsumoto, "Stress reduction and electric properties of InSb thin films grown by metalorganic vapor phase epitaxy on sapphire substrates with an InAs buffer layer," Vacuum, vol. 84, no. 11, pp. 1323-1326, 2010.

[22] T. Iwasugia, M. Moria, H. Igarashia, K. Murataa, M. Saitob, and K. Maezawa, "Heteroepitaxial growth of InSb films on the patterned Si (001) substrate," Physics Procedia, vol. 3, no. 2, pp. 1329-1333, 2010.

[23] J. A. A. Engelbrecht and M. C. Wagener, "Characterization of InSb layers on GaAs substrates using infrared reflectance and a modified oscillator formula," Physica B: Condensed Matter, vol. 404, no. 22, pp. 4397-4401, 2009.

[24] M. Li, Y. Qiu, G. Liu, Y. Wang, B. Zhang, and L. Zhao, "Distribution of dislocations in GaSb and InSb epilayers grown on GaAs (001) vicinal substrates," Journal of Applied Physics, vol. 105, no. 9, article 094903, 2009.

[25] B. T. Wysocki and M. A. Marciniak, "Discrimination between electronic and optical blooming in an InSb focal-plane array under high-intensity excitation," Infrared Physics \& Technology, vol. 51, no. 3, pp. 137-145, 2008.

[26] K. Yaemsunthorn, T. Thongtem, S. Thongtem, and C. Randorn, "Synthesis of InSb nanocrystals in an air atmosphere and their photocatalytic activity from near-infrared to ultra-violet," Materials Science in Semiconductor Processing, vol. 68, pp. 53-57, 2015. 
[27] T. Zhang, J. J. Harris, W. R. Branford, S. K. Clowes, L. F. Cohen, and S. A. Solin, "Exploration of the inherent magnetoresistance in InSb thin films," Semiconductor Science and Technology, vol. 21, no. 12, pp. 1543-1546, 2006.

[28] W. R. Branford, A. Husmann, S. A. Solin et al., "Geometric manipulation of the high-field linear magnetoresistance in InSb epilayers on GaAs (001)," Applied Physics Letters, vol. 86, no. 20, article 202116, 2005.

[29] I. Kimukin, N. Biyikli, and E. Ozbay, "InSb high-speed photodetectors grown on GaAs substrate," Journal of Applied Physics, vol. 94, no. 8, pp. 5414-5416, 2003.

[30] V. K. Dixita, B. V. Rodriguesa, H. L. Bhata, R. Venkataraghavanb, K. S. Chandrasekaranb, and B. M. Arorab, "Growth of InSb epitaxial layers on GaAs (001) substrates by LPE and their characterizations," Journal of Crystal Growth, vol. 235, no. 1-4, pp. 154-160, 2002.

[31] Z. C. Feng, C. Beckham, P. Schumaker et al., "Optical characterization and mapping of four inch InSb epitaxial thin films grown on GaAs by turbo disk metalorganic chemical vapor deposition," MRS Proceedings, vol. 450, pp. 61-66, 1996.

[32] K. Li, A. T. S. Wee, J. Lin et al., "A surface and interface study on the InSb/GaAs heterostructures," Thin Solid Films, vol. 302, no. 1-2, pp. 111-115, 1997.

[33] M. A. Abdulsattar, T. R. Sultan, and A. M. Saeed, "Shape and size dependence of electronic properties of InSb diamondoids and nanocrystals: a density functional theory study," Advances in Condensed Matter Physics, vol. 2013, Article ID 713267, 2013.

[34] Z. C. Feng, Q. X. Li, L. Y. Wan, and G. Xu, "Variation of phonon coupling factors in the photoluminescence of cadmium telluride by variable excitation power," Optical Materials Express, vol. 7, no. 3, pp. 808-816, 2017.

[35] S. Liu, X.-H. Zhao, C. M. Campbell, M. B. Lassise, Y. Zhao, and Y.-H. Zhang, "Carrier lifetimes and interface recombination velocities in $\mathrm{CdTe} / \mathrm{MgxCd} 1-x \mathrm{Te}$ double heterostructures with different $\mathrm{Mg}$ compositions grown by molecular beam epitaxy," Applied Physics Letters, vol. 107, no. 4, article 041120, 2015.

[36] C.-J. Wu, Z.-C. Feng, W.-M. Chang, C.-C. Yang, and H.-H. Lin, "Bond lengths and lattice structure of InP0.52Sb0.48 grown on GaAs," Applied Physics Letters, vol. 101, no. 9, article 091902, 2012.

[37] N. Yamamoto, K. Akahane, S.-I. Gozu, A. Ueta, and M. Tsuchiya, "Low-temperature growth of nanostructured InGaSb semiconductors on silicon substrates," Physica E: Low-Dimensional Systems and Nanostructures, vol. 40, no. 6, pp. 2195-2197, 2008.

[38] G. Tsai, D. L. Wang, and H. H. Lin, "Photoluminescence of In $\mathrm{As}_{0.04} \mathrm{P}_{0.67} \mathrm{Sb}_{0.29}$," Journal of Applied Physics, vol. 104, no. 2, article $023535,2008$.

[39] S. A. Cripps, T. J. C. Hosea, A. Krier et al., "Midinfrared photoreflectance study of InAs-rich InAsSb and GaInAsPSb indicating negligible bowing for the spin orbit splitting energy," Applied Physics Letters, vol. 90, no. 17, article 172106, 2017.

[40] M. Shafa, S. Akbar, L. Gao, M. Fakhar-e-Alam, and Z. M. Wang, "Indium antimonide nanowires: synthesis and properties," Nanoscale Research Letters, vol. 11, no. 1, p. 164, 2016.

[41] D. Wang and L. M. Tang, "Electronic structure and magnetism of doped wurtzite InSb nanowire," Journal of Physics D: Applied Physics, vol. 49, no. 17, article 175303, 2016.

[42] C. C. Ahiaa, N. Tile, Z. N. Urgessa, J. R. Botha, and J. H. Neethling, "An investigation of near-infrared photoluminescence from AP-MOVPE grown InSb/GaSb quantum dot structures," Journal of Crystal Growth, vol. 458, pp. 53-59, 2017.

[43] K. Sugiyama, "Molecular beam epitaxy of InSb films on CdTe," Journal of Crystal Growth, vol. 60, no. 2, pp. 450-452, 1982.

[44] R. Venkataraghavan, K. S. R. K. Rao, M. S. Hegde, and H. L. Bhat, "Influence of growth parameters on the surface and interface quality of laser deposited InSb/CdTe heterostructures," Physica Status Solidi (a), vol. 163, no. 1, pp. 93100, 1997.

[45] Z. C. Feng, S. Perkowitz, T. S. Rao, and J. B. Webb, "Resonance Raman scattering from epitaxial InSb films grown by metalorganic magnetron sputtering," Journal of Applied Physics, vol. 68, no. 10, pp. 5363-5365, 1990.

[46] M. C. Debnath, T. Zhang, C. Roberts, L. F. Cohen, and R. A. Stradling, "High-mobility InSb thin films on GaAs (001) substrate grown by the two-step growth process," Journal of Crystal Growth, vol. 267, no. 1-2, pp. 17-21, 2004.

[47] M. A. McKee, B.-S. Yoo, and R. A. Stall, "Uniform growth of $\mathrm{InSb}$ on GaAs in a rotating disk reactor by LP-MOVPE," Journal of Crystal Growth, vol. 124, no. 1-4, pp. 286-291, 1992.

[48] G. E. Jellison Jr. and F. A. Modine, "Parameterization of the optical functions of amorphous materials in the interband region," Applied Physics Letters, vol. 69, no. 3, pp. 371-373, 1996.

[49] B.-S. Yoo, M. A. McKee, S.-G. Kim, and E.-H. Lee, "Structural and electrical properties of InSb epitaxial films grown on GaAs by low-pressure MOCVD," Solid State Communications, vol. 88, no. 6, pp. 447-450, 1993.

[50] E. Woelk, H. Jurgensen, R. Rolph, and T. Zielinski, "Large scale production of indium antimonide film for position sensors in automobile engines," Journal of Electronic Materials, vol. 24, no. 11, pp. 1715-1718, 1995.

[51] A. A. M. Farag, F. S. Terra, G. M. Mahmoud, and A. M. Mansour, "Study of Gaussian distribution of inhomogeneous barrier height for n-InSb/p-GaAs heterojunction prepared by flash evaporation," Journal of Alloys and Compounds, vol. 481, no. 1-2, pp. 427-433, 2009.

[52] S. Adachi, Optical Constants of Crystalline and Amorphous Semiconductors Numerical Data and Graphical Information, Springer, Berlin, Germany, 1999, ISBN: 9780792385677.

[53] T. J. Kim, S. Y. Hwang, J. S. Byun et al., "Temperature dependence of the dielectric functions and the critical points of InSb by spectroscopic ellipsometry from 31 to 675K," Journal of Applied Physics, vol. 114, no. 10, article 103501, 2013. 


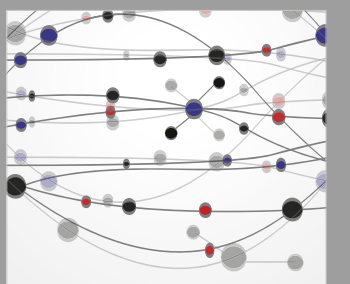

The Scientific World Journal
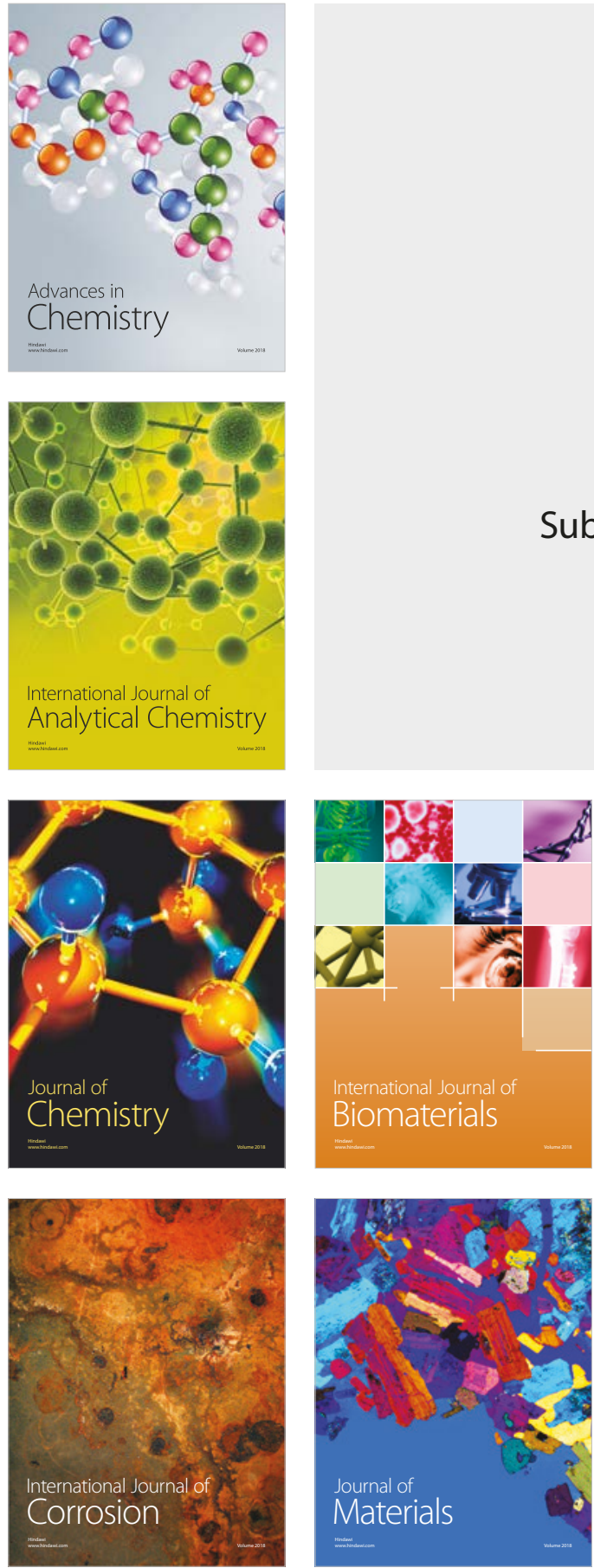

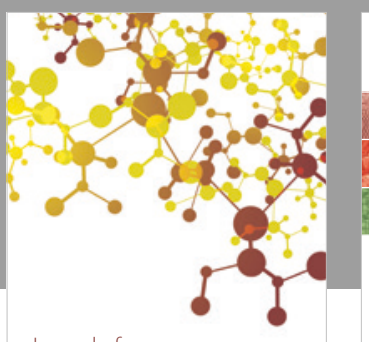

Journal of

Applied Chemistry
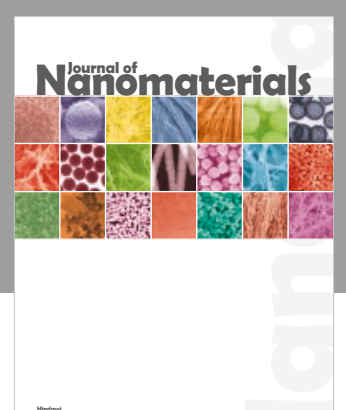

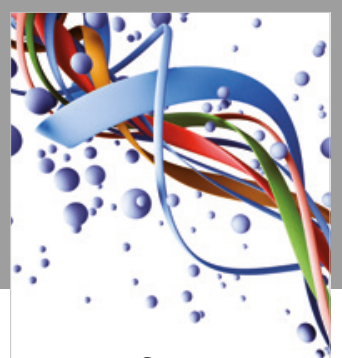

Scientifica

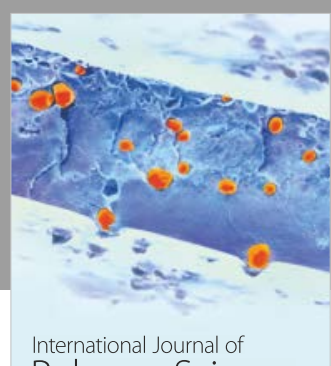

Polymer Science

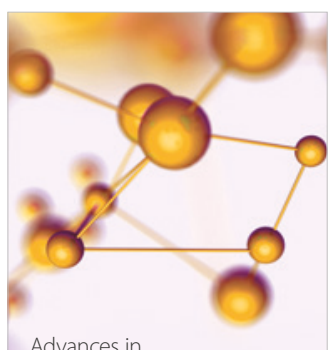

Physical Chemistry
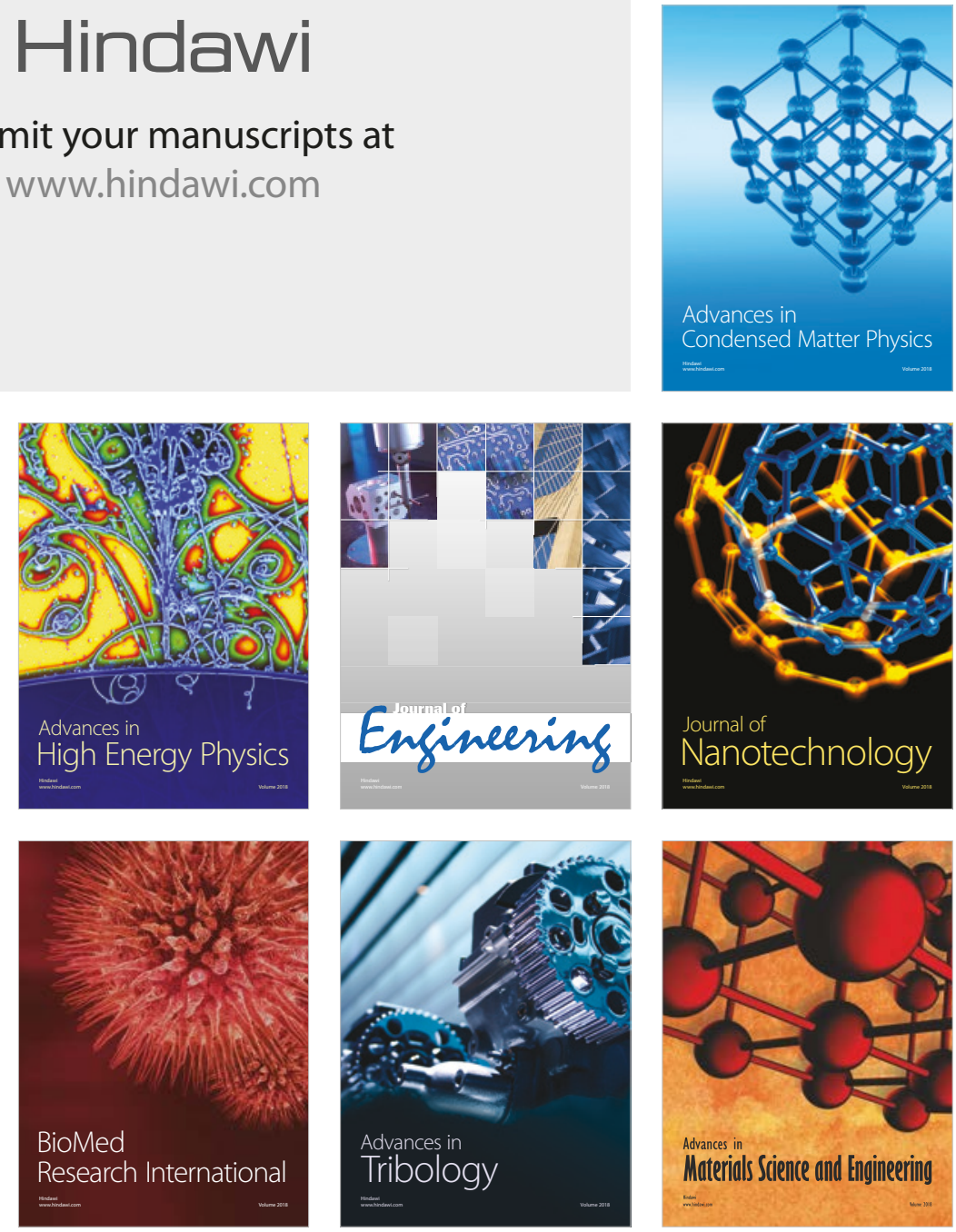